\title{
ON STARLIKENESS AND CLOSE-TO-CONVEXITY OF CERTAIN ANALYTIC FUNCTIONS
}

\section{OH SANG KWON and NAK EUN CHO}

\author{
Received 20 January 2004
}

\begin{abstract}
Our purpose is to derive some sufficient conditions for starlikeness and close-to-convexity of order $\alpha$ of certain analytic functions in the open unit disk.
\end{abstract}

2000 Mathematics Subject Classification: 30C45.

1. Introduction. Let $A_{n}$ be the class of functions of the form

$$
f(z)=z+\sum_{k=n+1}^{\infty} a_{k} z^{k} \quad(n \in \mathbb{N}=\{1,2,3, \ldots\})
$$

which are analytic in the open unit disk $U=\{z:|z|<1\}$. A function $f \in A_{n}$ is said to be in the class $S_{n}^{*}(\alpha)$ if it satisfies

$$
\operatorname{Re}\left\{\frac{z f^{\prime}(z)}{f(z)}\right\}>\alpha \quad(z \in U)
$$

for some $\alpha(0 \leq \alpha<1)$. A function in the class $S_{n}^{*}(\alpha)$ is starlike of order $\alpha$ in $U$. We also write $A_{1}=A$ and $S_{1}^{*}(\alpha)=S^{*}(\alpha)$.

Let $C_{n}(\alpha)$ be the subclass of $A_{n}$ consisting of functions $f(z)$ which satisfy

$$
\operatorname{Re}\left\{f^{\prime}(z)\right\}>\alpha \quad(z \in U)
$$

for some $\alpha(0 \leq \alpha<1)$. A function $f(z)$ in $C_{n}(\alpha)$ is close-to-convex of order $\alpha$ in $U$ (cf. Duren [1]).

Let $f(z)$ and $g(z)$ be analytic in $U$. Then the function $f(z)$ is said to be subordinate to $g$, written $f \prec g$ or $f(z) \prec g(z)$, if there exists an analytic function $w(z)$ with $w(0)=0$ and $|w(z)|<1(z \in U)$ such that $f(z)=g(w(z))$ for $z \in U$. If $g(z)$ is univalent in $U$, then $f(z) \prec g(z)$ is equivalent to $f(0)=g(0)$ and $f(U) \subset g(U)$.

Let $H\left(p(z), z p^{\prime}(z)\right) \prec h(z)$ be a first-order differential subordination. Then a univalent function $q(z)$ is called its dominant if $p(z) \prec q(z)$ for all analytic functions $p(z)$ that satisfy the differential subordination. A dominant $\bar{q}(z)$ is called the best dominant if $\bar{q}(z) \prec q(z)$ for all dominants $q(z)$. For the general theory of first-order differential subordination and its applications, we refer to [3].

Recently, $\mathrm{Xu}$ and Yang [5] obtained some results on starlikeness and close-toconvexity of certain meromorphic functions. In the present note, we investigate some 
sufficient conditions for starlikeness and close-to-convexity of order $\alpha$ of certain analytic functions in $U$ by using the subordination principle, and obtain some useful corollaries as special cases. Furthermore, we extend the results given by Owa et al. [4].

2. Main results. To derive our results, we need the following lemmas.

LEMMA 2.1 [6]. Let $g(z)=b_{0}+b_{n} z^{n}+b_{n+1} z^{n+1}+\cdots(n \in \mathbb{N})$ be analytic in $U$ and let $h(z)$ be analytic and starlike (with respect to the origin), univalent in $U$ with $h(0)=0$. If $z g^{\prime}(z) \prec h(z)(z \in U)$, then

$$
g(z) \prec b_{0}+\frac{1}{n} \int_{0}^{z} \frac{h(t)}{t} d t .
$$

LEMMA 2.2 [3]. Let $g(z)$ be analytic and univalent in $U$ and let $\theta(w)$ and $\varphi(w)$ be analytic in a domain $D$ containing $g(U)$, with $\varphi(w) \neq 0$ when $w \in g(U)$. Set

$$
Q(z)=z g^{\prime}(z) \varphi(g(z)), \quad h(z)=\theta(g(z))+Q(z)
$$

and suppose that

(i) $Q(z)$ is univalent and starlike in $U$;

(ii) $\operatorname{Re}\left\{z h^{\prime}(z) / Q(z)\right\}=\operatorname{Re}\left\{\theta^{\prime}(g(z)) / \varphi(g(z))+z Q^{\prime}(z) / Q(z)\right\}>0(z \in U)$. If $p(z)$ is analytic in $U$, with $p(0)=g(0), p(U) \subset D$, and

$$
\theta(p(z))+z p^{\prime}(z) \varphi(p(z)) \prec \theta(g(z))+z g^{\prime}(z) \varphi(g(z))=h(z),
$$

then $p(z) \prec g(z)$ and $g(z)$ is the best dominant of (2.3).

LEMMA 2.3 [2]. Let $g(z)=b_{0}+b_{n} z^{n}+b_{n+1} z^{n+1}+\cdots(n \in \mathbb{N})$ be analytic in $U$ with $g(z) \not \equiv b_{0}$. If $0<\left|z_{0}\right|<1$ and $\operatorname{Re}\left\{g\left(z_{0}\right)\right\}=\min _{|z| \leq\left|z_{0}\right|} \operatorname{Re}\{g(z)\}$, then

$$
z_{0} g^{\prime}\left(z_{0}\right) \leq-\frac{n\left|b_{0}-g\left(z_{0}\right)\right|^{2}}{2 \operatorname{Re}\left\{b_{0}-g\left(z_{0}\right)\right\}} .
$$

Applying Lemma 2.1, we now derive the following.

THEOREM 2.4. Let $f \in A_{n}$ satisfy $f(z) f^{\prime}(z) \neq 0$ for $z \in U \backslash\{0\}$ and

$$
-\alpha \frac{z f^{\prime}(z)}{f(z)}+\frac{z f^{\prime \prime}(z)}{f^{\prime}(z)}+\alpha \prec \frac{a z}{1-b z} \quad(z \in U),
$$

where $\alpha, a$, and $b$ are real numbers with $a \neq 0$ and $b \leq 1$.

(i) If $0<a \leq n$ and $0<b \leq 1$, then

$$
\operatorname{Re}\left\{\frac{z^{\alpha} f^{\prime}(z)}{f^{\alpha}(z)}\right\}>\left(\frac{1}{1+b}\right)^{a / n b} \quad(z \in U) .
$$

(ii) If $0<a \leq n$ and $b=0$, then

$$
\operatorname{Re}\left\{\frac{z^{\alpha} f^{\prime}(z)}{f^{\alpha}(z)}\right\}>e^{-a / n} \quad(z \in U) .
$$


(iii) If $a \neq 0$ and $0<b \leq 1$, then

$$
\left|\left(\frac{z^{\alpha} f^{\prime}(z)}{f^{\alpha}(z)}\right)^{-n b / a}-1\right|<b \quad(z \in U) .
$$

(iv) If $a>0$ and $b=0$, then

$$
\left|\frac{z^{\alpha} f^{\prime}(z)}{f^{\alpha}(z)}-1\right|<e^{a / n}-1 \quad(z \in U) .
$$

Proof. Let $f \in A_{n}$ with $f(z) f^{\prime}(z) \neq 0$ for $z \in U \backslash\{0\}$ and define

$$
g(z)=-\alpha\left(\frac{z f^{\prime}(z)}{f(z)}-1\right)+\frac{z f^{\prime \prime}(z)}{f^{\prime}(z)} .
$$

Then $g(z)=b_{n} z^{n}+b_{n+1} z^{n+1}+\cdots$ is analytic in $U$ and (2.5) can be rewritten as

$$
g(z) \prec h(z)
$$

where $h(z)=a z /(1-b z)$ is analytic and starlike in $U$. Applying Lemma 2.1 to (2.11), we have

$$
\int_{0}^{z} \frac{g(t)}{t} d t \prec \frac{1}{n} \int_{0}^{z} \frac{h(t)}{t} d t
$$

that is,

$$
-\alpha \int_{0}^{z}\left(\frac{f^{\prime}(t)}{f(t)}-\frac{1}{t}\right) d t+\int_{0}^{z} \frac{f^{\prime \prime}(t)}{f^{\prime}(t)} d t \prec \frac{a}{n} \int_{0}^{z} \frac{d t}{1-b t}
$$

(i) If $0<a \leq n$ and $0<b \leq 1$, then from (2.13) we deduce that

$$
\frac{z^{\alpha} f^{\prime}(z)}{f^{\alpha}(z)} \prec\left(\frac{1}{1-b z}\right)^{a / n b} \equiv h_{1}(z)
$$

The function $h_{1}(z)$ is analytic and convex univalent in $U$ because

$$
\operatorname{Re}\left\{1+\frac{z h_{1}^{\prime \prime}(z)}{h_{1}(z)}\right\}=\operatorname{Re}\left\{\frac{1+(a / n) z}{1-b z}\right\} \geq \frac{1-a / n}{1+b} \geq 0 \quad(z \in U) .
$$

Also, $h_{1}(U)$ is symmetric with respect to the real axis. Hence $\operatorname{Re}\left\{h_{1}(z)\right\}>h_{1}(-1)$ in $U$ and it follows from (2.14) that

$$
\operatorname{Re}\left\{\frac{z^{\alpha} f^{\prime}(z)}{f^{\alpha}(z)}\right\}>\left(\frac{1}{1+b}\right)^{a / n b} \quad(z \in U) .
$$

(ii) If $0<a \leq n$ and $b=0$, then from (2.13) we obtain

$$
\frac{z^{\alpha} f^{\prime}(z)}{f^{\alpha}(z)} \prec e^{(a / n) z} \equiv h_{2}(z)
$$


Since $h_{2}(z)$ is analytic and convex univalent in $U$ and $h_{2}(U)$ is symmetric with respect to the real axis, it follows from (2.17) that

$$
\operatorname{Re}\left\{\frac{z^{\alpha} f^{\prime}(z)}{f^{\alpha}(z)}\right\}>e^{-a / n} \quad(z \in U) .
$$

(iii) If $a \neq 0$ and $0<b \leq 1$, then by (2.14) we have

$$
\frac{z^{\alpha} f^{\prime}(z)}{f^{\alpha}(z)}=\left(\frac{1}{1-b w(z)}\right)^{a / n b} \quad(z \in U),
$$

where $w(z)$ is analytic in $U$ with $|w(z)| \leq|z|(z \in U)$. Therefore we have

$$
\left|\left(\frac{z^{\alpha} f^{\prime}(z)}{f^{\alpha}(z)}\right)^{-n b / a}-1\right|<|-b w(z)|<b \quad(z \in U) .
$$

(iv) If $a>0$ and $b=0$, then from (2.17) we get

$$
\frac{z^{\alpha} f^{\prime}(z)}{f^{\alpha}(z)}=e^{(a / n) w(z)} \quad(z \in U),
$$

where $w(z)$ is analytic in $U$ with $|w(z)| \leq|z|(z \in U)$. Thus

$$
\left|\frac{z^{\alpha} f^{\prime}(z)}{f^{\alpha}(z)}-1\right|=\left|e^{(a / n) w(z)}-1\right| \leq e^{(a / n)|w(z)|}-1<e^{a / n}-1 \quad(z \in U) .
$$

Therefore the proof of Theorem 2.4 is completed.

By specifying the values of the parameters appearing in Theorem 2.4, we can obtain several useful corollaries.

Taking $0<a=2(\alpha-\beta) \leq n$ and $b=1$, Theorem 2.4(i) reduces to the following.

COROLlary 2.5. Let $f \in A_{n}$ satisfy $f(z) f^{\prime}(z) \neq 0$ for $z \in U \backslash\{0\}$ and

$$
\operatorname{Re}\left\{\alpha \frac{z f^{\prime}(z)}{f(z)}-\frac{z f^{\prime \prime}(z)}{f^{\prime}(z)}\right\}<2 \alpha-\beta \quad(z \in U),
$$

where $\alpha$ is a real number and $\alpha-n / 2 \leq \beta<\alpha$, then

$$
\operatorname{Re}\left\{\frac{z^{\alpha} f^{\prime}(z)}{f^{\alpha}(z)}\right\}>\frac{1}{2^{(2(\alpha-\beta) / n)}} \quad(z \in U) .
$$

REMARK 2.6. Owa et al. [4] proved that if $f \in A_{n}$ satisfies $f(z) f^{\prime}(z) \neq 0$ for $z \in$ $U \backslash\{0\}$ and (2.23) for $\alpha \geq 0$ and $\alpha-n / 2 \leq \beta<\alpha$, then

$$
\operatorname{Re}\left\{\frac{z^{\alpha} f^{\prime}(z)}{f^{\alpha}(z)}\right\}>\frac{n}{n+2 \alpha-2 \beta} \quad(z \in U) .
$$

In view of $2^{x}<1+x(0<x<1)$, Corollary 2.5 is better than the main theorem of [4].

COROLlarY 2.7. If $f \in A_{n}$ satisfies $f(z) f^{\prime}(z) \neq 0$ for $z \in U \backslash\{0\}$ and

$$
\operatorname{Re}\left\{\frac{z f^{\prime}(z)}{f(z)}-\frac{z f^{\prime \prime}(z)}{f^{\prime}(z)}\right\}<1+\frac{a}{2} \quad(z \in U)
$$

for some $a(0<a \leq n)$, then $f \in S_{n}^{*}\left(2^{-a / n}\right)$ and the order $2^{-a / n}$ is sharp. 
Proof. Letting $\alpha=b=1$ in Theorem 2.4(i) and using (2.26), we see that $f \in S_{n}^{*}\left(2^{-a / n}\right)$. To show that the order $2^{-a / n}$ cannot be increased, we consider

$$
f(z)=\exp \int_{0}^{z} \frac{\left(1+t^{n}\right)^{-a / n}}{t} d t \in A_{n} .
$$

It is easy to verify that the function $f(z)$ defined by (2.27) satisfies (2.26) and

$$
\operatorname{Re}\left\{\frac{z f^{\prime}(z)}{f(z)}\right\}=\operatorname{Re}\left\{\left(\frac{1}{1+z^{n}}\right)^{a / n}\right\} \rightarrow\left(\frac{1}{2}\right)^{a / n}
$$

as $z \rightarrow 1$. Therefore the proof is completed.

Putting $\alpha=0$ and $b=1$ in Theorem 2.4(i), we have the following.

COROLlary 2.8. If $f \in A_{n}$ satisfies $f^{\prime}(z) \neq 0$ for $z \in U \backslash\{0\}$ and

$$
-\operatorname{Re}\left\{\frac{z f^{\prime \prime}(z)}{f^{\prime}(z)}\right\}<\frac{a}{2} \quad(z \in U)
$$

for some $a(0<a \leq n)$, then $f \in C_{n}\left(2^{-a / n}\right)$ and the order $2^{-a / n}$ is sharp.

REMARK 2.9. Corollary 2.7 (with $0<a=2(1-\beta) \leq n$ ) and Corollary 2.8 (with $0<$ $a=2 \beta<n)$ are better than the corresponding results in [4].

Setting $\alpha=0$ and 1 in Theorem 2.4(ii), we have the following two corollaries.

Corollary 2.10. If $f \in A_{n}$ satisfies $f(z) f^{\prime}(z) \neq 0$ for $z \in U \backslash\{0\}$ and

$$
\left|\frac{z f^{\prime \prime}(z)}{f^{\prime}(z)}\right|<a \quad(z \in U)
$$

for some $a(0<a \leq n)$, then $f \in C_{n}\left(e^{-a / n}\right)$.

Corollary 2.11. If $f \in A_{n}$ satisfies $f(z) f^{\prime}(z) \neq 0$ for $z \in U \backslash\{0\}$ and

$$
\left|1+\frac{z f^{\prime \prime}(z)}{f^{\prime}(z)}-\frac{z f^{\prime}(z)}{f(z)}\right|<a \quad(z \in U)
$$

for some $a(0<a \leq n)$, then $f \in S_{n}^{*}\left(e^{-a / n}\right)$ and the order $e^{-a / n}$ is sharp with the extremal function

$$
f(z)=\exp \int_{0}^{z} \frac{e^{-(a / n) t^{n}}}{t} d t
$$

For $\alpha=1$ and $a=-n b(0<b \leq 1)$ in Theorem 2.4(iii), we have the following.

Corollary 2.12. If $f \in A_{n}$ satisfies $f(z) f^{\prime}(z) \neq 0$ for $z \in U \backslash\{0\}$ and

$$
1+\frac{z f^{\prime \prime}(z)}{f^{\prime}(z)}-\frac{z f^{\prime}(z)}{f(z)} \prec-\frac{-n b z}{1-b z}
$$

for some $b(0<b \leq n)$, then $f \in S_{n}^{*}(1-b)$ and the order $1-b$ is sharp with the extremal function $f(z)=z e^{(b / n) z^{n}}$. 
Next, applying Lemma 2.2, we obtain the following two results.

THEOREM 2.13. Let $f \in A$ satisfy $f(z) \neq 0$ for $z \in U \backslash\{0\}$ and

$$
\frac{z f^{\prime}(z)}{f(z)}+\frac{z^{2} f^{\prime \prime}(z)}{f(z)} \prec h(z) \quad(z \in U),
$$

where

$$
h(z)=\frac{(1-2 \alpha)^{2} z^{2}+2(2-3 \alpha)+1}{(1-z)^{2}} \quad(0 \leq \alpha<1 ; z \in U)
$$

then $f \in S^{*}(\alpha)$ and the order $\alpha$ is sharp.

Proof. We put

$$
\frac{z f^{\prime}(z)}{f(z)}=(1-\alpha) p(z)+\alpha
$$

for $0 \leq \alpha<1$. Then $p(z)$ is analytic in $U$ and $p(0)=1$. Differentiating (2.36) logarithmically, we find that

$$
\frac{z f^{\prime}(z)}{f(z)}+\frac{z^{2} f^{\prime \prime}(z)}{f(z)}=(1-\alpha) z p^{\prime}(z)+((1-\alpha) p(z)+\alpha)^{2}
$$

From (2.34) and (2.37), we have

$$
(1-\alpha) z p^{\prime}(z)+(1-\alpha)^{2} p^{2}(z)+2 \alpha(1-\alpha) p(z)+\alpha^{2} \prec h(z) .
$$

Now we choose

$$
g(z)=\frac{1+z}{1-z}, \quad \theta(w)=(1-\alpha)^{2} w^{2}+2(1-\alpha) w+\alpha^{2}, \quad \varphi(w)=1-\alpha .
$$

Then $g(z)$ is analytic and univalent in $U, \operatorname{Re}\{g(z)\}>0(z \in U)$, and $\theta(w)$ and $\varphi(w)$ are analytic with $\varphi(w) \neq 0$ in the $w$-plane.

The function

$$
Q(z)=z g^{\prime}(z) \varphi(z)=2(1-\alpha) \frac{z}{(1-z)^{2}}
$$

is univalent and starlike in $U$. Further,

$$
\begin{aligned}
\theta(g(z))+Q(z) & =(1-\alpha)^{2}\left(\frac{1+z}{1-z}\right)^{2}+2 \alpha(1-\alpha)\left(\frac{1+z}{1-z}\right)+\alpha^{2}+2(1-\alpha) \frac{z}{1-z} \\
& =\frac{(1-2 \alpha)^{2} z^{2}+2(2-3 \alpha) z+1}{(1-z)^{2}}=h(z), \\
\operatorname{Re}\left\{\frac{z h^{\prime}(z)}{Q(z)}\right\} & =\operatorname{Re}\left\{2(1-\alpha) g(z)+2 \alpha+\frac{z Q^{\prime}(z)}{Q(z)}\right\} \\
& =(3-2 \alpha) \operatorname{Re}\left\{\frac{1+z}{1-z}\right\}+2 \alpha>0
\end{aligned}
$$


for $z \in U$. In view of (2.38)-(2.42), we see that

$$
\theta(p(z))+z p^{\prime}(z) \varphi(p(z)) \prec \theta(g(z))+z g^{\prime}(z) \varphi(g(z))=h(z) .
$$

Therefore, Lemma 2.2 leads to $p(z) \prec g(z)$, which implies that $f \in S^{*}(\alpha)$. Next, we consider

$$
f(z)=\frac{z}{(1-z)^{2(1-\alpha)}} \in A .
$$

It is easy to see that

$$
\begin{gathered}
\frac{z f^{\prime}(z)}{f(z)}+\frac{z^{2} f^{\prime \prime}(z)}{f(z)}=h(z), \\
\operatorname{Re}\left\{\frac{z f^{\prime}(z)}{f(z)}\right\}=\operatorname{Re}\left\{\frac{1+(1-2 \alpha) z}{1-z}\right\} \rightarrow \alpha
\end{gathered}
$$

as $z \rightarrow-1$. The proof of the theorem is completed.

THEOREM 2.14. If $f \in A$ satisfies $f(z) \neq 0$ for $z \in U \backslash\{0\}$ and

$$
\frac{z f^{\prime}(z)}{f(z)}+2 \alpha \frac{z^{2} f^{\prime \prime}(z)}{f(z)} \prec h(z),
$$

where

$$
h(z)=\frac{(2 \alpha-1)^{3} z^{2}+2 \alpha(3-4 \alpha) z+1}{(1-z)^{2}} \quad(0 \leq \alpha<1 ; z \in U),
$$

then $f \in S^{*}(\alpha)$ and the order $\alpha$ is sharp.

Proof. It suffices to prove the theorem for $0<\alpha<1$. We define the function $p(z)$ by (2.36). Then $p(z)$ is analytic in $U$ and $p(0)=1$. By a simple calculation, we find that

$$
\begin{aligned}
& \frac{z f^{\prime}(z)}{f(z)}+2 \alpha \frac{z^{2} f^{\prime \prime}(z)}{f(z)} \\
& \quad=2 \alpha(1-\alpha) z p^{\prime}(z)+2 \alpha(1-\alpha)^{2} p^{2}(z)+(1-\alpha)\left(1-2 \alpha+4 \alpha^{2}\right) p(z) \\
& \quad+\alpha\left(1-2 \alpha+2 \alpha^{2}\right) .
\end{aligned}
$$

Thus the subordination (2.46) becomes

$$
\begin{aligned}
& 2 \alpha(1-\alpha) z p^{\prime}(z)+2 \alpha(1-\alpha)^{2} p^{2}(z)+(1-\alpha)\left(1-2 \alpha+4 \alpha^{2}\right) p(z) \\
& \quad+\alpha\left(1-2 \alpha+2 \alpha^{2}\right) \prec h(z) .
\end{aligned}
$$

Set $g(z)=(1+z) /(1-z), \theta(w)=2 \alpha(1-\alpha)^{2} w^{2}+(1-\alpha)\left(1-2 \alpha+4 \alpha^{2}\right) w+\alpha(1-2 \alpha+$ $2 \alpha^{2}$ ), and $\varphi(w)=2 \alpha(1-\alpha)$. Then $g(z), \theta(w)$, and $\varphi(w)$ satisfy the conditions of Lemma 2.2. The function

$$
Q(z)=z g^{\prime}(z) \varphi(g(z))=4 \alpha(1-\alpha) \frac{z}{(1-z)^{2}}
$$


is univalent and starlike in $U$. Further,

$$
\begin{aligned}
\theta(g(z))+Q(z)= & 2 \alpha(1-\alpha)^{2}\left(\frac{1+z}{1-z}\right)^{2}+(1-\alpha)\left(1-2 \alpha+4 \alpha^{2}\right)\left(\frac{1+z}{1-z}\right) \\
& +\alpha\left(1-2 \alpha+2 \alpha^{2}\right)+4 \alpha(1-\alpha) \frac{z}{(1-z)^{2}} \\
= & \frac{(2 \alpha-1)^{3} z^{2}+2 \alpha(3-4 \alpha) z+1}{(1-z)^{2}}=h(z), \\
\operatorname{Re}\left\{\frac{z h^{\prime}(z)}{Q(z)}\right\}= & \operatorname{Re}\left\{2(1-\alpha) g(z)+\frac{1-2 \alpha+4 \alpha^{2}}{2 \alpha}+\frac{z Q^{\prime}(z)}{Q(z)}\right\} \\
= & (3-2 \alpha) \operatorname{Re}\left\{\frac{1+z}{1-z}\right\}+\frac{1-2 \alpha+4 \alpha^{2}}{2 \alpha}>0,
\end{aligned}
$$

for $z \in U$. Note that

$$
\theta(p(z))+z p^{\prime}(z) \varphi(p(z)) \prec \theta(g(z))+z g^{\prime}(z) \varphi(g(z))=h(z) .
$$

Hence, an application of Lemma 2.2 yields that $p(z) \prec g(z)$, that is, $f \in S^{*}(\alpha)$. For the function $f(z)$ defined by (2.44), we have

$$
\begin{gathered}
\frac{z f^{\prime}(z)}{f(z)}+2 \alpha \frac{z^{2} f^{\prime \prime}(z)}{f(z)}=h(z), \\
\operatorname{Re}\left\{\frac{z f^{\prime}(z)}{f(z)}\right\} \rightarrow \alpha \quad \text { as } \quad z \longrightarrow-1 .
\end{gathered}
$$

Therefore we complete the proof of Theorem 2.14.

Finally, by using Lemma 2.3, we prove the following.

THEOREM 2.15. Let $f \in A_{n}$ satisfy $f(z) \neq 0$ for $z \in U \backslash\{0\}$ and

$$
\left|\arg \left\{(1-\lambda) \frac{z^{2}\left(f^{\prime}(z)\right)^{2}}{f^{2}(z)}+\lambda\left(\frac{z f^{\prime}(z)}{f(z)}+\frac{z^{2} f^{\prime \prime}(z)}{f(z)}\right)+\frac{n \lambda}{2}\right\}\right|<\pi \quad(z \in U)
$$

for some $\lambda(\lambda>0)$. Then $f \in S_{n}^{*}(0)$ and the order 0 is sharp.

Proof. The function $g(z)$ defined by

$$
g(z)=\frac{z f^{\prime}(z)}{f(z)}=1+b_{n} z^{n}+b_{n+1} z^{n+1}+\cdots
$$

is analytic in $U$ and it is easily verified that

$$
(1-\lambda) \frac{z^{2}\left(f^{\prime}(z)\right)^{2}}{f^{2}(z)}+\lambda\left(\frac{z f^{\prime}(z)}{f(z)}+\frac{z^{2} f^{\prime \prime}(z)}{f(z)}\right)=g^{2}(z)+\lambda z g^{\prime}(z) \quad(\lambda>0 ; z \in U) .
$$

Suppose that there exists a point $z_{0} \in U \backslash\{0\}$ such that

$$
\operatorname{Re}\{g(z)\}>0 \quad\left(|z|<\left|z_{0}\right|\right), \quad g\left(z_{0}\right)=i \beta,
$$


where $\beta$ is a real number. Then, applying Lemma 2.3 , we have

$$
z_{0} g^{\prime}\left(z_{0}\right) \leq-\frac{n\left(1+\beta^{2}\right)}{2}
$$

Thus it follows from (2.56), (2.57), and (2.58) that

$$
\begin{aligned}
(1-\lambda) & \frac{z_{0}^{2}\left(f^{\prime}\left(z_{0}\right)\right)^{2}}{f^{2}\left(z_{0}\right)}+\lambda\left(\frac{z f^{\prime}\left(z_{0}\right)}{f\left(z_{0}\right)}+\frac{z_{0}^{2} f^{\prime \prime}\left(z_{0}\right)}{f\left(z_{0}\right)}\right)+\frac{n \lambda}{2} \\
& =\left(g\left(z_{0}\right)\right)^{2}+\lambda z_{0} g^{\prime}\left(z_{0}\right)+\frac{n \lambda}{2} \\
& \leq-\beta^{2}-\frac{n \lambda\left(1+\beta^{2}\right)}{2}+\frac{n \lambda}{2} \leq 0
\end{aligned}
$$

for $\lambda>0$, which contradicts (2.54). Hence $\operatorname{Re}\{g(z)\}>0(z \in U)$, that is $f \in S_{n}^{*}(0)$. If we let

$$
f_{n}(z)=\frac{z}{\left(1-z^{n}\right)^{2 / n}} \in A_{n}
$$

then

$$
\begin{gathered}
(1-\lambda) \frac{z^{2}\left(f_{n}^{\prime}(z)\right)^{2}}{f_{n}^{2}(z)}+\lambda\left(\frac{z f_{n}^{\prime}(z)}{f_{n}(z)}+\frac{z^{2} f_{n}^{\prime \prime}(z)}{f_{n}(z)}\right)+\frac{n \lambda}{2} \\
=\left(1+\frac{n \lambda}{2}\right)\left(\frac{1+z^{n}}{1-z^{n}}\right)^{2} \quad(z \in U),
\end{gathered}
$$

and so the function $f_{n}(z)$ satisfies (2.54). Noting that

$$
\operatorname{Re} \frac{z f_{n}^{\prime}(z)}{f_{n}(z)}=\operatorname{Re} \frac{1+z^{n}}{1-z^{n}} \longrightarrow 0
$$

as $z \rightarrow e^{i \pi / n}$, we conclude that the order 0 is the best possible.

ACKNOWLEDGMENT. The authors would like to express their gratitude to the referee for his valuable suggestions.

\section{REFERENCES}

[1] P. L. Duren, Univalent Functions, Grundlehren der Mathematischen Wissenschaften, vol. 259, Springer-Verlag, New York, 1983.

[2] S. S. Miller and P. T. Mocanu, Second-order differential inequalities in the complex plane, J. Math. Anal. Appl. 65 (1978), no. 2, 289-305.

[3] _ On some classes of first-order differential subordinations, Michigan Math. J. 32 (1985), no. 2, 185-195.

[4] S. Owa, M. Nunokawa, H. Saitoh, and S. Fukui, Starlikeness and close-to-convexity of certain analytic functions, Far East J. Math. Sci. 2 (1994), no. 2, 143-148.

[5] N. Xu and D. Yang, On starlikeness and close-to-convexity of certain meromorphic functions, J. Korea Soc. Math. Educ. Ser. B Pure Appl. Math. 10 (2003), no. 1, 1-11. 
[6] D. Yang, On sufficient conditions for multivalent starlikeness, Bull. Korean Math. Soc. 37 (2000), no. 4, 659-668.

Oh Sang Kwon: Department of Mathematics, Kyungsung University, Pusan 608-736, Korea E-mail address: oskwon@star. kyungsung. ac. kr

Nak Eun Cho: Department of Applied Mathematics, Pukyong National University, Pusan 608737, Korea

E-mail address: necho@pknu.ac.kr 


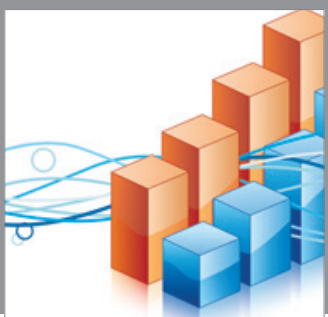

Advances in

Operations Research

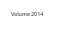

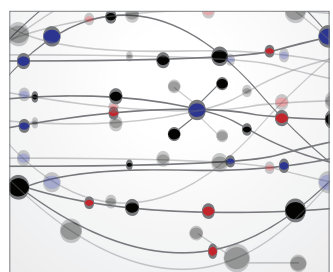

\section{The Scientific} World Journal
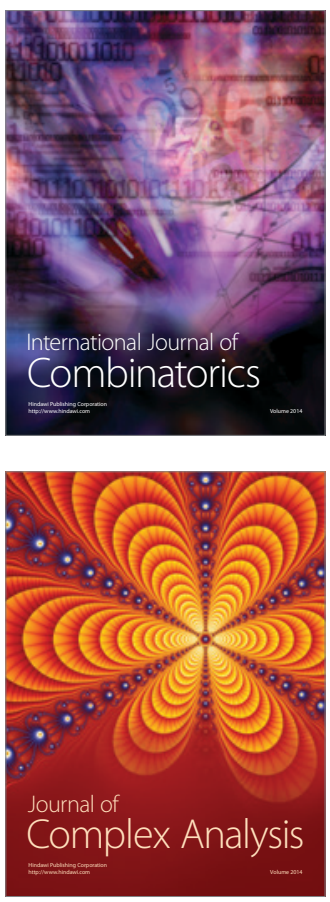

International Journal of

Mathematics and

Mathematical

Sciences
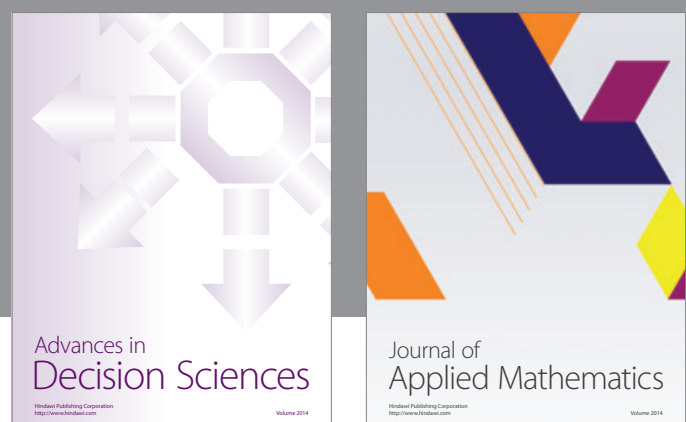

Journal of

Applied Mathematics
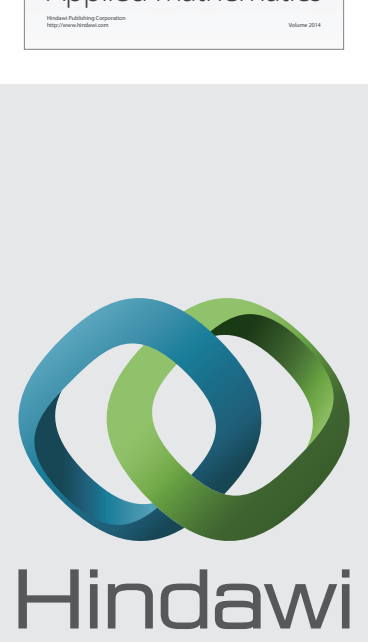

Submit your manuscripts at http://www.hindawi.com
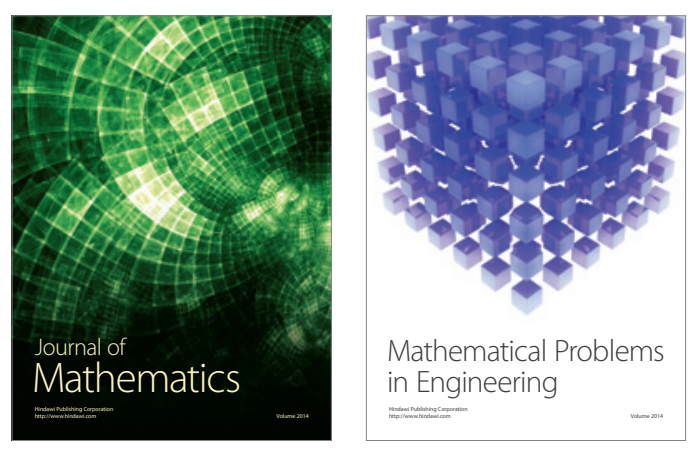

Mathematical Problems in Engineering
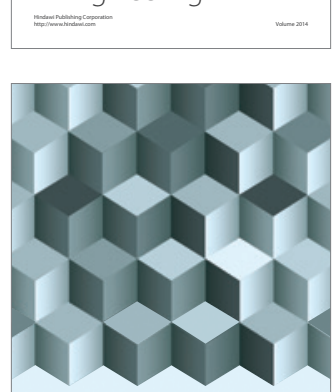

Journal of

Function Spaces
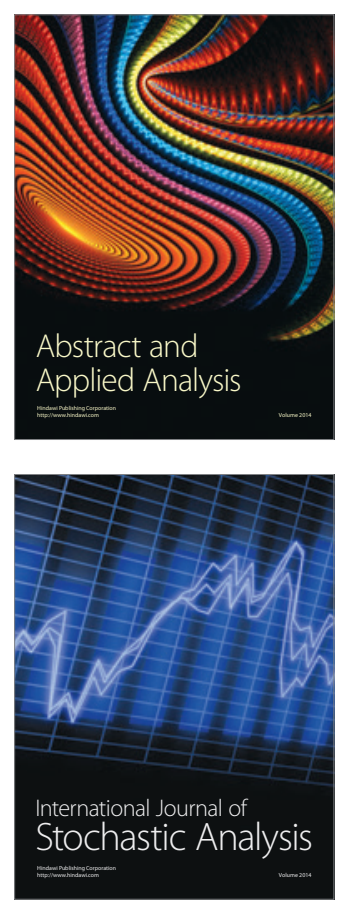

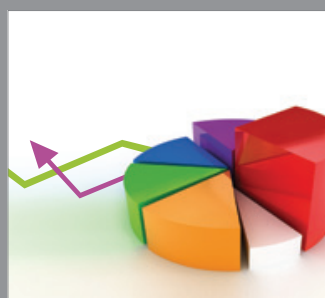

ournal of

Probability and Statistics

Promensencen
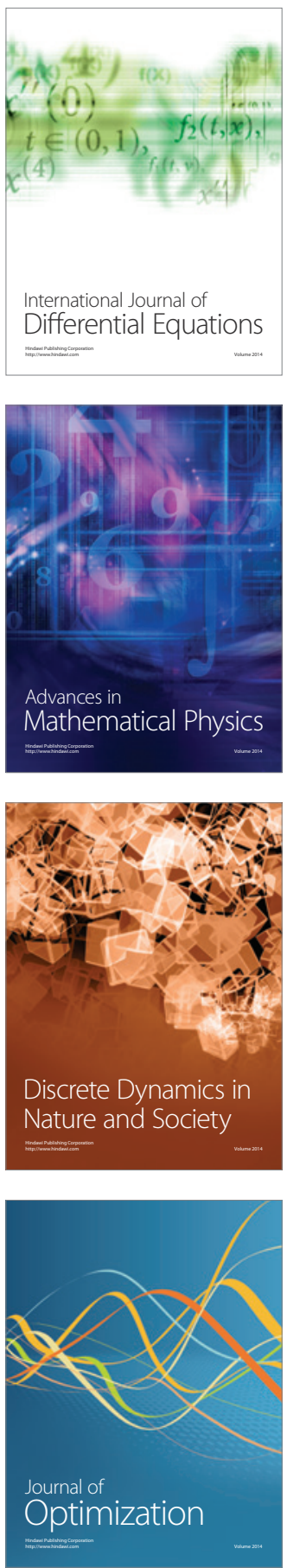